\title{
Lessons of the month 1: Shoshin beriberi: A case report of fulminant cardiovascular collapse, intractable hyperlactatemia and deteriorating consciousness
}

\author{
Authors: Marie SH Lim, ${ }^{A}$ Wutyee Win, ${ }^{B}$ Anna Von Essen, ${ }^{C}$ David Gannon ${ }^{D}$ and Mohamed Ramali ${ }^{\mathrm{E}}$
}

Shoshin beriberi is a fulminant variant of thiamine deficiency, often presenting with severe lactic acidosis and cardiogenic shock. Due to the sparsity of this condition, delays in diagnosis can lead to fatality. However, rapid reversal of symptoms can be easily achieved through intravenous thiamine replacement.

In this case report, we discuss a 57-year-old woman, who was previously fit and well, who presented to the emergency department with a 3-day history of extreme malaise, breathlessness and abdominal pain, with marked hypotension and tachycardia requiring vasopressor support and a severe rising lactic acidosis. Upon further questioning, a history of alcohol excess was noted. Rapid reversal of the marked haemodynamic instability was achieved upon administration of intravenous thiamine and the patient was discharged within 48 hours.

KEYWORDS: Shoshin beriberi, circulatory shock, lactataemia, thiamine deficiency, beriberi

DOI: $10.7861 /$ clinmed.2021-0550

\section{Introduction}

Thiamine deficiency (beriberi) is a nutritional disease that is associated with various states of poor nutrition, such as alcohol excess. ${ }^{1,2}$ Shoshin beriberi is a fulminant variant characterised by cardiogenic shock and lactic acidosis and can have fatal consequences. ${ }^{3}$ Despite this, it is a highly treatable condition with favourable outcomes upon intravenous thiamine administration. Here, we report a case of Shoshin beriberi presenting with severe lactic acidosis and cardiovascular collapse requiring intensive care admission for inotropic support, with reattainment of haemodynamic stability within hours of intravenous thiamine administration.

Authors: ${ }^{A}$ IMT2 trainee, Colchester General Hospital, Colchester,

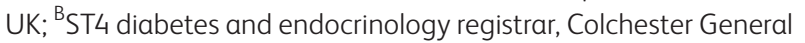

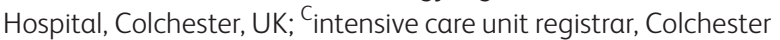
General Hospital, Colchester, UK; ${ }^{D}$ consultant acute physician, Colchester General Hospital, Colchester, UK; Eintensive care unit consultant, Colchester General Hospital, Colchester, UK

\section{Case presentation}

A 57-year-old woman was admitted to the emergency department with 3 days of extreme malaise, breathlessness and generalised abdominal pain. She noted unquantified weight loss over an extended period of time. She was well and mobile 1 week prior to admission, with no significant past medical history and not requiring any regular medications.

On examination, she appeared cachectic. Pulse rate was raised at 111 beats per minute, with a blood pressure of $80 / 47 \mathrm{mmHg}$ and capillary refill time of longer than 3 seconds. Respiratory rate was elevated at 30 breaths per minute; however, examination of chest expansion and lung fields had no significant abnormality. Tympanic temperature was $36.6^{\circ} \mathrm{C}$. The abdomen was soft and non-tender, with no organomegaly. Glasgow coma score was 15 with a normal neurological exam.

An initial arterial blood gas on room air demonstrated a severe metabolic acidosis with respiratory compensation (Table 1; Fig 1). Subsequent blood tests demonstrated significant neutrophilia with monocytosis, with deranged liver function tests (Table 2 ).

Imaging performed showed an unremarkable chest $\mathrm{X}$-ray and abdominal computed tomography.

Given the elevated white cell count with neutrophilia, a working diagnosis of sepsis was made, with standard broad-spectrum antibiotics and intravenous fluid resuscitation initiated.

\section{Table 1. Arterial blood gas results on admission}

$\begin{array}{lll}\text { Test performed } & \text { Result } & \text { Reference value } \\ \mathrm{pH} & 6.90 & 7.35-7.45 \\ \begin{array}{l}\text { Partial pressure of } \\ \text { carbon dioxide, } \mathrm{kPa}\end{array} & 1.28 & 4.7-6.0 \\ \begin{array}{l}\text { Partial pressure of } \\ \text { oxygen, } \mathrm{kPa}\end{array} & 14.4 & 11.3-14.0 \\ \begin{array}{l}\text { Bicarbonate, } \mathrm{mmol} / \mathrm{L} \\ \text { Base excess, } \mathrm{mmol} / \mathrm{L}\end{array} & 4.8 & 22-30 \\ \text { Lactate, } \mathrm{mmol} / \mathrm{L} & -30 & -2-2 \\ \text { Calculated anion gap, } & 18 & 0.5-2.2 \\ \mathrm{mEq} / \mathrm{L} & 19.3 & \end{array}$



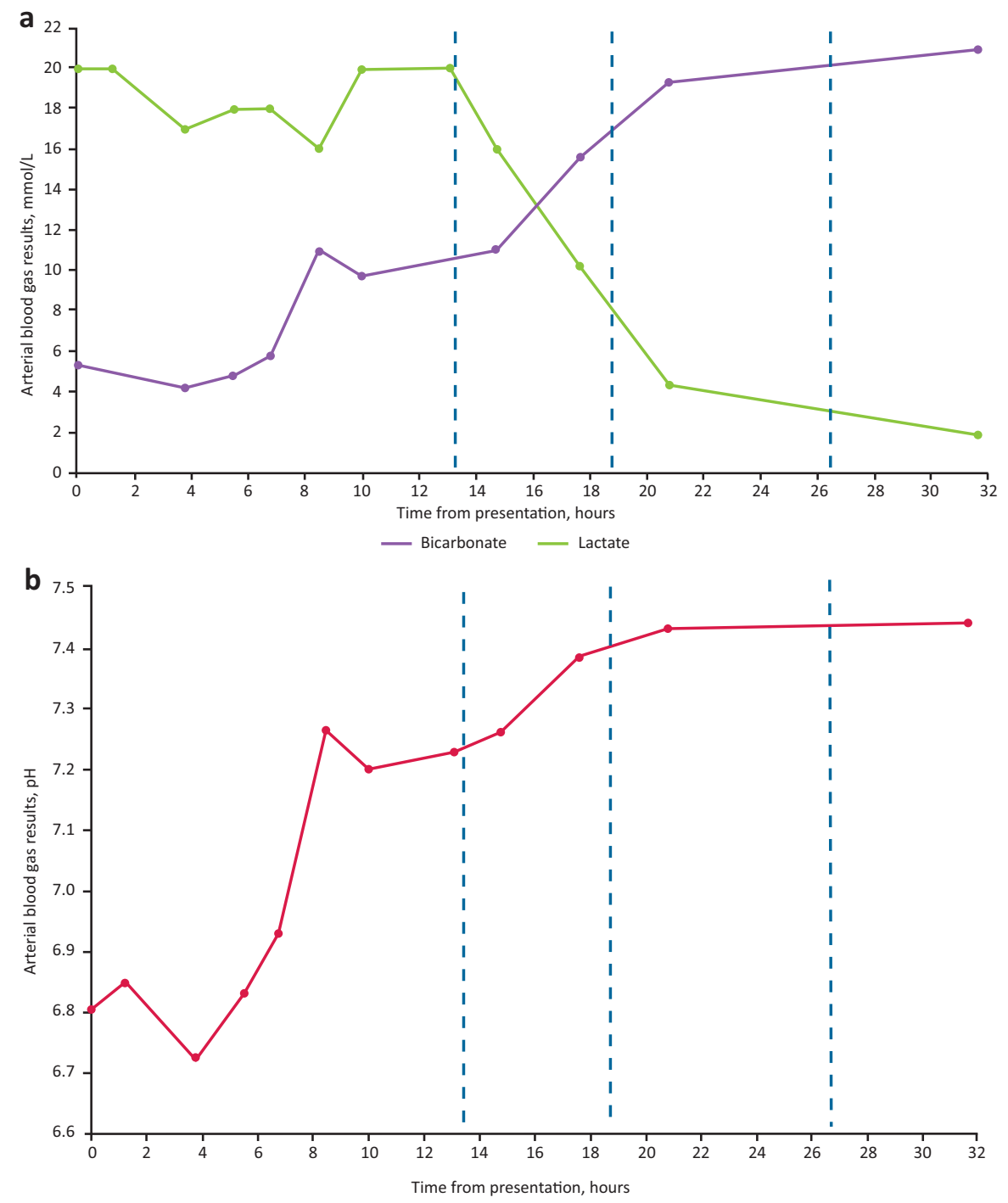

Fig 1. Arterial blood gases after thiamine administration. a) Lactate and bicarbonate levels. b) pH levels. Blue dotted lines denote when thiamine was administered.

However, as the blood pressure did not improve following 6 hours of fluid resuscitation, the patient was transferred to intensive care for inotropic support. Additionally, a 1.26\% bicarbonate infusion at $500 \mathrm{~mL} /$ hour was commenced for 2 hours; this was subsequently reduced to $200 \mathrm{~mL} /$ hour. A metaraminol infusion at $0.3 \mathrm{~mL} /$ hour was also commenced. The urinary output was measured as consistently over $100 \mathrm{~mL} /$ hour.

A repeat arterial blood gas performed showed improvement in the metabolic acidosis; however, an increase in the serum lactate was noted (Fig 1).

Further questioning yielded an extensive history of alcohol excess of 56 units a week, along with a diet consisting almost exclusively of biscuits and chocolates. A working diagnosis of Shoshin beriberi was made. $500 \mathrm{mg}$ of intravenous thiamine was therefore administered; after 2 hours, hemodynamic stability was achieved and inotropic support discontinued. Repeat arterial blood gas 4 hours after thiamine administration demonstrated a normal pH with improving serum lactate (Fig 1).

The patient was stepped down from intensive care after observations overnight, with a further 24 hours of monitoring, before being subsequently discharged with a lifelong prescription of $100 \mathrm{mg}$ thiamine daily. At 2 weeks' follow-up, continued alcohol abstinence and dietary improvement was noted.

\section{Discussion}

Beriberi has traditionally been classified into two main types: dry beriberi or wet beriberi. The former is characterised by peripheral neuropathy, with the severity correlating to the extent and duration of thiamine deficiency; the latter typically presents with symptoms of right-sided heart failure (such as dyspnoea and peripheral oedema). A fulminant variant of both types, termed Shoshin beriberi, can present with cardiogenic shock, lactic acidosis and subsequently progress to multi-organ failure and death, if untreated. ${ }^{3}$ The development of lactic acidosis is due to the impact of thiamine deficiency on aerobic metabolism and the Krebs cycle, with subsequent inhibition causing rising levels of substrates, such as pyruvate and lactate. ${ }^{4}$

Beriberi was originally described by Aalsmeer and Wenkebach in Asia in 1929 and linked to diets rich in polished white rice. ${ }^{5}$ 
Table 2. Results of blood tests on admission

$\begin{array}{lll}\text { Test performed } & \text { Result } & \text { Reference value } \\ \text { Haemoglobin, } \mathrm{g} / \mathrm{L} & 133 & 130-180 \\ \text { White cell count, } \times 10^{9} / \mathrm{L} & 15.5 & 4-11 \\ \text { Neutrophils, } \times 10^{9} / \mathrm{L} & 12.9 & 2-7.5 \\ \text { Monocytes, } \times 10^{9} / \mathrm{L} & 1.2 & 0.2-1 \\ \text { Prothrombin time, seconds } & 13.1 & 10-15 \\ \text { Activated partial thrombin } & 30.7 & 25-36 \\ \text { time, seconds } & & \\ \text { Sodium, mmol/L } & 136 & 133-146 \\ \text { Potassium, mmol/L } & 4.7 & 3.5-5.3 \\ \text { Creatinine, } \mu \mathrm{mol} / \mathrm{L} & 72 & 45-84 \\ \text { Urea, } \mathrm{mmol} / \mathrm{L} & 1.4 & 2.5-7.8 \\ \text { Albumin, } \mathrm{g} / \mathrm{L} & 35 & 35-50 \\ \text { Bilirubin, } \mu \mathrm{mol} / \mathrm{L} & 40 & 0-21 \\ \text { Alkaline phosphatase, } \mathrm{U} / \mathrm{L} & 172 & 30-130 \\ \text { Alanine aminotransferase, } & 217 & 0-41 \\ \text { U/L } & & \\ & & \end{array}$

However, by the 1960s the disease was in decline due to improved nutrition, health propaganda and heightened public awareness. ${ }^{6}$ While less commonly seen in developed countries, beriberi is associated with states of poor nutritional intake (such as alcohol excess, dietary zealots and the elderly). ${ }^{7}$

By extension, Shoshin beriberi was considered a rare disease in developed countries. ${ }^{2}$ However, there have been multiple reports of isolated cases in recent years; the main contributing factors of which were alcohol excess, use of total parenteral nutrition without vitamin supplementation or chronic malnutrition. ${ }^{1,2,8-10}$ Likewise to the case presented here, a profound lactic acidosis was noted upon presentation in the other cases mentioned, with the onset of rapid onset congestive cardiac failure occurring prior to or during hospitalisation. A rising lactate and high vasopressor requirements were other common features.

However, given the dramatic and severe presentation of Shoshin beriberi, the management for Shoshin beriberi is surprisingly straightforward: intravenous administration of thiamine. Indeed, largely favourable outcomes with rapid reversal of symptoms and haemodynamic stability within hours were noted upon intravenous thiamine administration, findings that were similarly observed in this case. ${ }^{1,2,8}$

While every effort should be made to measure serum thiamine levels, this may not always be feasible given the rapidity of symptom progression; Shoshin beriberi can be fatal if not managed promptly. ${ }^{9}$ However, it should be noted that the presentation of Shoshin beriberi mimics that of several more common diseases, such as sepsis or cardiac failure. A high clinical suspicion should therefore be maintained when managing such cases that do not appear to be responding to classic therapies combined with a thorough social history, if available, are the key means of establishing a diagnosis of Shoshin beriberi, which as illustrated in this case, can be managed in a simple and effective manner with prompt results.

\section{References}

1 Kountchev J, Bijuklic K, Bellmann R, Joannidis M. A patient with severe lactic acidosis and rapidly evolving multiple organ failure: a case of shoshin beri-beri. Intensive Care Med 2005;31:1004.

2 Attas M, Hanley HG, Stultz D, Jones MR, McAllister RG. Fulminant beriberi heart disease with lactic acidosis: presentation of a case with evaluation of left ventricular function and review of pathophysiologic mechanisms. Circulation 1978;58:566-72.

3 Daroff RB. Bradley's neurology in clinical practice e-book. Elsevier, 2015:2845.

4 Dabar G, Harmouche C, Habr B, Riachi M, Jaber B. Shoshin beriberi in critically-ill patients: case series. Nutr ] 2015;14:51.

5 Aalsmeer WC, Wenckebach KF. [Heart and circulation in beriberi]. Wien Arch Inn Med 1929:16:193.

6 Arnold D. British India and the "Beriberi Problem", 1798-1942. Med Hist 2010;54:295-314.

7 Finglas PM. Thiamin. Int ] Vitam Nutr Res 1993;63:270-4.

8 Chadda K, Raynard B, Antoun S, Thyrault M, Nitenberg G. Acute lactic acidosis with Wernicke's encephalopathy due to acute thiamine deficiency. Intensive Care Med 2002;28:1499.

9 Bello S, Neri M, Riezzo I et al. Cardiac beriberi: morphological findings in two fatal cases. Diagn Pathol 2011;6:8.

10 Vicinanza A, De Laet C, Rooze $S$ et al. Shoshin beriberi and severe accidental hypothermia as causes of heart failure in a 6-year-old child: a case report and brief review of literature. Front Pediatr 2019;7:119.

Address for correspondence: Dr Marie Sok Hui Lim, Colchester General Hospital, Turner Road, Colchester CO4 5JL, UK.

Email: marie.lim@doctors.org.uk 